\title{
Clinico-Demographic Profile of Vaccinated/Partially Vaccinated Versus Non-Vaccinated Covid-19 Cases in Second Wave of India
}

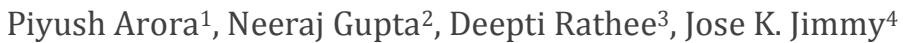 \\ 1,2,4 Department of Respiratory Medicine, Jawaharlal Nehru Medical College and Hospital, Ajmer, Rajasthan, India. \\ ${ }^{3}$ Department of Pulmonary and Critical Care Medicine, Mittal Hospital and Research Centre, Ajmer, Rajasthan, India.
}

\section{ABSTRACT}

\section{BACKGROUND}

As of 20th November 2021, $29.1 \%$ (40.1 crore) of the population has been completely vaccinated while $55.6 \%$ (76.6 crore) of the population have received at least one dose of vaccine. Infection despite vaccination (vaccine breakthrough) has been reported, but characteristics of these infection regarding clinico demographic profile, severity of disease and mortality in the fully vaccinated, partially vaccinated and non-vaccinated groups are not well described; during the second Covid-19 wave in India from April to June 2021 when the highly transmissible delta variant predominated.

\section{METHODS}

This was an observational analysis of epidemiological features, clinical manifestations and outcome of Covid-19 positive patients during the second wave. Vaccination status, CT scoring, RT PCR reports, bio markers like CRP, d dimer, IL-6 were recorded. Statistical analysis was done using Epi Info version 7.2.1.0 statistical software. The study was approved by institutional research and ethical committee.

\section{RESULTS}

182 Covid patients had received only one dose, 24 were fully vaccinated and 24 were unvaccinated. Unvaccinated patients were younger than vaccinated people ( $p$ $<0.001$ ) CT severity scoring were relatively higher in unvaccinated patients as compared to vaccinated patients. $(\mathrm{p}=0.003)$. $92.3 \%$ of our vaccinated patients' group were treated on OPD basis while in unvaccinated patients $41.7 \%$ required hospitalisation. Biomarkers (CRP, D-DIMER and IL-6) were also deranged to a lesser severity among vaccinated and partially vaccinated study population as compared to unvaccinated individuals. Death occurred in $8.3 \%$ of unvaccinated patients and only $1.1 \%$ of partially vaccinated patients, while no mortality occurred in fully vaccinated patients

\section{CONCLUSIONS}

We can conclude that vaccines play a critical role in preventing serious Covid-19 illness and remain highly effective in preventing Covid-19 hospitalizations and the biomarkers (CRP, d dimer, IL-6) can be used in the risk stratification of Covid-19 infections

\section{KEY WORDS}

Second Covid-19 Wave in India, Delta Variant, Vaccine Breakthrough Infections, Hospitalisations, Bio Markers, D Dimer, CRP, IL-6.
Corresponding Author:

Dr. Jose K. Jimmy,

$3^{\text {rd }}$ Year Junior Resident,

Department of Respiratory Medicine, Jawaharlal Nehru Medical College \&

Hospital, Ajmer, Rajasthan, India.

E-mail: josekjimmy3124@gmail.com

DOI: $10.14260 / j e m d s / 2022 / 23$

How to Cite This Article:

Arora P, Gupta N, Rathee D, et al. Clinico demographic profile of vaccinated/ partially vaccinated versus non-vaccinated Covid 19 cases in second wave of India. J Evolution Med Dent Sci 2022;11(01):120125, DOI: $10.14260 / \mathrm{jemds} / 2022 / 23$

Submission 14-12-2021,

Peer Review 21-12-2021,

Acceptance 17-01-2022,

Published 28-01-2022.

Copyright (c) 2022 Piyush Arora et al. This is an open access article distributed under Creative Commons Attribution License [Attribution 4.0 International (CC BY 4.0)] 


\section{BACKGROUND}

Severe acute respiratory syndrome coronavirus-2 (SARSCoV-2) was reported from Wuhan in December 2019 and rapidly spread across the globe. The World Health Organization (WHO) declared it as a Public Health Emergency of International Concern on 11th March, 2020. The Covid-19 pandemic in India is a part of the worldwide pandemic of coronavirus disease 2019. As of 20 November 2021, according to official figures, India has the second-highest number of confirmed cases in the world (after the United States) with 34.5 million reported cases of Covid19 infection and the third-highest number of Covid-19 deaths (after the United States and Brazil) at 4.66 lakh deaths. However, these figures probably exhibit severe under-reporting. ${ }^{1,2}$

The first case of Covid-19 disease in India was reported on 27 January 2020 and the first wave took off at the end of March 2020. On 12 September 2020, the highest number of cases during first wave was recorded $(97,570)$. During the first week of February 2021, the lowest number of new cases were noted. ${ }^{3}$ However, by early-April 2021, a major second wave of infections took hold in the country with destructive consequences, ${ }^{5}$ on 9 April, India surpassed 1 million active cases, ${ }^{6}$ and by 12 April, India overtook Brazil as having the second-most Covid-19 cases worldwide. ${ }^{7}$ By late April, India passed 2.5 million active cases and was reporting an average of 300,000 new cases and 2,000 deaths per-day. Some analysts feared this was an undercount. ${ }^{8}$ On 30 April, India reported over 400,000 new cases and over 3,500 deaths in one day

Vaccines are considered the mainstay in halting and ending the Covid-19 pandemic. The vaccination programme was started in India on 16 January 2021. The vaccination drive was launched in a phased manner. Beginning with healthcare, sanitation and essential frontline workers, followed by the geriatric population, people with comorbidities, those aged $>45$ years and finally the entire adult population. ${ }^{4}$

\section{Phase 1}

Started on 16 January 2021 and targeted 10 million health workers first followed by 20 million frontline workers. Phase 1 was to be completed by 31 March.

\section{Phase 2}

Began on 1 March 2021 to cover 45+ year old with comorbidities and 60+ year old. On 1 April, vaccinations were opened for everyone above 45 years. Shortages in vaccine supplies were evident in March 2021

\section{Phase 3}

Vaccination campaign was opened up to include all eligible adults (18+) from 1 May 2021 following a surge in cases in April, a second wave. This expansion resulted in immediate, increased and prolonged vaccine shortages. $9,10,11$

The vaccines approved and utilized in india by the regulatory authority included AZD1222 - ChAdOx1-S (Covishield), manufactured in India by Serum Institute of India through license from Astrazeneca-Oxford ${ }^{3}$ and BBV152 (Covaxin), indigenous vaccine developed by Bharat Biotech in association with the Indian Council of Medical Research
(ICMR). The approval of Covaxin was met with some concern, as the vaccine had not then completed phase 3 trials. Due to this status, those receiving Covaxin were required to sign a consent form, while some states chose to relegate Covaxin to a "buffer stock" and primarily distribute the OxfordAstraZeneca vaccine. Following the conclusion of its trial, the DCGI issued a standard emergency use authorisation to Covaxin in March 2021.12,13

The AZD122 (ChAdOx1-S/nCoV-19) recombinant vaccine against Covid-19 is a replication-deficient adenoviral vector vaccine that expresses the SARS-CoV-2 spike protein gene. BBV152 is a whole-virion inactivated SARS-CoV-2 vaccine adjuvanted with Algel-IMDG to induce T-helper-1 cell (Th1) responses. ${ }^{14}$ The efficacy of AZD1222 (ChAdOx1-S) after administration of two doses of the vaccines irrespective of interval between the doses has been reported as $63.1 \%$, with possibly higher efficacy on longer intervals. ${ }^{14}$

The interim phase- 3 clinical trial data reported BBV152 to have efficacy of $78 \%$ against infection with SARS-CoV- 2.15 However, the real-world effectiveness of vaccines may differ from the efficacy reported in clinical trials due to a multitude of factors including the dynamics of disease exposure, diminished antibody response in sub-groups like the elderly and the immunocompromised, and the emergence of newer mutant strains with greater infectivity and virulence.

A small percentage of vaccinated persons will develop symptomatic or asymptomatic infections with SARS-CoV-2, as no vaccine accords $100 \%$ protection against the disease, and occasionally newer virus variants evolve mechanisms for bypassing the vaccine induced antibody response. A vaccine breakthrough infection is defined as the detection of SARSCoV-2 RNA or antigen in a respiratory specimen collected from a person $\geq 14$ days after receipt of recommended doses of Covid-19 vaccine

According to the ICMR between 0.02 and $0.04 \%$ infections have occurred after partial or complete vaccination with either BBV152 or AZD1222 (ChAdOx1-S). ${ }^{16}$

The first mutation in the virus was discovered in the spike-protein (D614G) leading to increased infectivity. ${ }^{17}$ Other newSARS-CoV-2 variants of concern (VOC) i.e., Alpha (B.1.1.7), Beta (B.1.351, B.1.351.2, B.1.351.3) ${ }^{18}$ and Gamma (P.1, P.1.1, P.1.2) were detected from United Kingdom, South Africa and Brazil respectively during September to December 2020 and subsequently were reported from India. The spread of these variants globally, worsened the Covid-19 pandemic with increased transmissibility, enhanced severity of illness, lowered response to vaccines and monoclonal antibodies. ${ }^{19}$ The emergence of the delta variant (B.1.617.2, AY.1, AY.2, AY.3) can be attributed to the devastating second wave in India

The effectiveness and protection developed by the vaccines have been doubted in the light of development of variants of concern and reduced effectiveness of certain candidate vaccines against these variants of concern. Israel reported breakthrough Covid-19 infections in individuals immunized with the Pfizer vaccine on 9th April, 2021.20 During April 2021, Hacisuleyman et al. reported 417 cases of breakthrough infections in individuals vaccinated with Pfizer and Moderna mRNA vaccines. ${ }^{21}$

Centre for Disease Prevention and Control (CDC), reported a total of 10,262 Covid-19 vaccine breakthrough infections. ${ }^{22}$ Breakthrough infections in healthcare workers 
vaccinated with the BNT162b2 vaccine were reported from Italy in the month of May, 2021 during an outbreak of SARSCoV-2 lineage B.1.1.7.23 In India, few studies reported breakthrough infections in small parts of the country like Kerala $^{24}$ and Delhi. ${ }^{25}$ Considering these reports, this study was undertaken to identify the clinicodemographic profile of Covid-19 cases in vaccinated / partially vaccinated individuals and compare them with new covid 19 cases.

\section{Objectives}

1. To assess the clinico demographic profile of vaccinated versus partially vaccinated versus non vaccinated patients of Covid-19 during second wave in India.

2. To assess the severity of Covid infection in all these three categories of patients with reference to CT severity score and laboratory markers

3. To assess the time interval between last dose of vaccination and date of onset of symptoms

4. To assess the mortality rates in these three groups of patients.

\section{METHODS}

This was an observational analysis of epidemiological features, clinical manifestations and outcome of Covid-19 positive patients during the second wave in Northern India from April 20, 2021 to June 30, 2021.

All laboratory or clinically confirmed cases were included in this study and the epidemiological, clinical, and laboratory data were recorded from bedside tickets, face to face interviews in OPD and through telephonic calls.

Age, sex, residence, co-morbidities, symptoms of all patients were recorded by personal interview with the patients infected with Covid-19 during second wave. CT scorings ${ }^{26}$ and RT PCR reports were also recorded. Markers of severity of infection or inflammation like CRP, D dimer, IL6 were also recorded at the time of presentation. Patients were also interviewed regarding their place of treatment for the infection, whether it was indoor, outdoor or day care treatment.

Vaccination status of all the patients were recorded by the help of interview with the patient and patients relative as well as confirmed by co-win app.

\section{Statistical Analysis}

Categorical variables were expressed as frequency and percentage and were analysed using chi square test. A p value $\leq 0.05$ was taken as statistically significant. All statistical analysis was done using Epi Info version 7.2.1.0 statistical software.

The study was approved by the institutional research and ethical committee.

\section{RESULTS}

A total of 182 Covid patients had received only single dose of vaccine, 24 Covid patients had received both doses of vaccination while 24 were unvaccinated. Unvaccinated patients were relatively younger as compared to vaccinated people $(\mathrm{p}<0.001)$. Those with co morbidities had higher chances of contracting Covid infection irrespective of vaccination status. Unvaccinated patients were relatively younger as compared to vaccinated people $(\mathrm{p}<0.001)$ (Table 1). No significant difference was seen for most symptoms, except cough $(\mathrm{p}<0.05)$ and haemoptysis $(\mathrm{p}<0.001)$, which were significantly more in unvaccinated patients. CTSS were relatively higher in unvaccinated patients as compared to vaccinated patients $(\mathrm{p}=0.003)$. Most patients with complete vaccination (92.3\%) and partial vaccination (84.6\%) required only OPD treatment, while indoor treatment was required in significantly higher patient $(41.7 \%)$ who were unvaccinated (Table 2). The level of the Covid specific inflammatory markers of severity (CRP, D Dimer \& IL-6) were relatively more elevated in unvaccinated patients and were least elevated in patient with both dose of vaccine (Table 3). The differences were statistically significant. Death occurred in $8.3 \%$ of unvaccinated patients and only $1.1 \%$ of partially vaccinated patients (Figure 1), while no mortality occurred in fully vaccinated patients (Table 4).

\begin{tabular}{|c|c|c|c|c|c|}
\hline & & $\begin{array}{l}\text { Unvaccinated } \\
\qquad(\mathrm{N}=24)\end{array}$ & $\begin{array}{c}\text { Single Dose } \\
(\mathrm{N}=182)\end{array}$ & $\begin{array}{c}\text { Both Dose } \\
(\mathrm{N}=26)\end{array}$ & $\mathbf{P}$ \\
\hline \multirow{5}{*}{$\begin{array}{c}\text { Age } \\
\text { (years) }\end{array}$} & $21-30$ & $0(0 \%)$ & $6(3.3 \%)$ & $0(0 \%)$ & \multirow{5}{*}{$\begin{array}{l}<0.001 \\
\quad(S)\end{array}$} \\
\hline & $31-40$ & $12(50 \%)$ & $22(12.1 \%)$ & $4(15.4 \%)$ & \\
\hline & $41-50$ & $8(33.3 \%)$ & $40(22 \%)$ & $10(38.5 \%)$ & \\
\hline & $51-60$ & $4(16.7 \%)$ & $84(46.2 \%)$ & $2(7.7 \%)$ & \\
\hline & $>60$ & $0(0 \%)$ & $30(16.5 \%)$ & $10(38.5 \%)$ & \\
\hline \multirow{2}{*}{ Residence } & Urban & $18(75 \%)$ & $160(87.9 \%)$ & $24(92.3 \%)$ & \multirow{2}{*}{0.146} \\
\hline & Rural & $6(25)$ & $22(12.1 \%)$ & $2(7.7 \%)$ & \\
\hline Any & Yes & $4(16.7 \%)$ & $98(53.8 \%)$ & $12(46.2 \%)$ & 0.003 \\
\hline comorb & No & $20(83.3 \%)$ & $84(46.2 \%)$ & $14(53.8 \%)$ & (S) \\
\hline \multirow{8}{*}{ 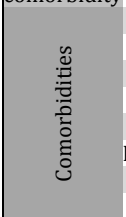 } & Asthma & $2(8.3 \%)$ & $14(7.7 \%)$ & $2(7.7 \%)$ & 0.994 \\
\hline & COPD & $0(0 \%)$ & $4(2.2 \%)$ & $0(0 \%)$ & 0.572 \\
\hline & CTD & $0(0 \%)$ & $2(1.1 \%)$ & $0(0 \%)$ & 0.758 \\
\hline & DM & $2(8.3 \%)$ & $50(27.5 \%)$ & $4(15.4 \%)$ & 0.065 \\
\hline & HTN & $2(830)$ & $52(28.6 \%)$ & $6(23.1 \%)$ & 0.098 \\
\hline & Hypothyroidism & $2(8.3 \%)$ & $15(8.2 \%)$ & $0(0 \%)$ & 0.345 \\
\hline & IHD & $0(0 \%)$ & $4(2.2 \%)$ & $0(0 \%)$ & 0.572 \\
\hline & CKD & $0(0 \%)$ & $2(1.1 \%)$ & $0(0 \%)$ & 0.758 \\
\hline
\end{tabular}

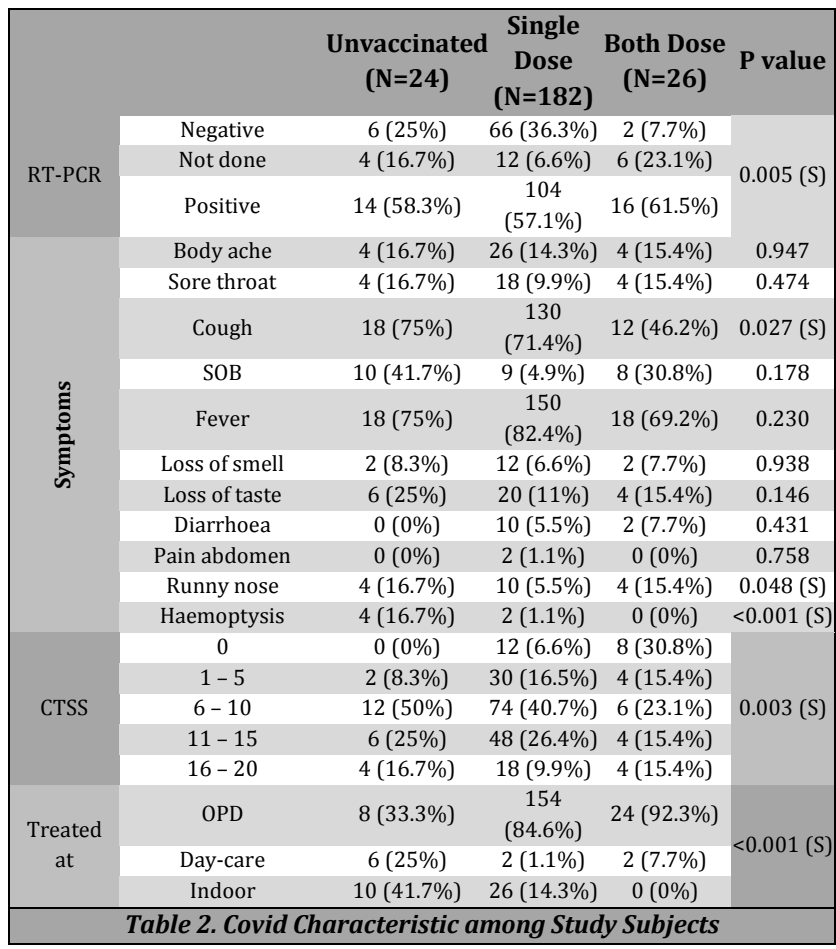




\begin{tabular}{|c|c|c|c|c|c|}
\hline & & $\begin{array}{l}\text { Unvaccinated } \\
\qquad(\mathrm{N}=24)\end{array}$ & $\begin{array}{c}\text { Single Dose } \\
(\mathrm{N}=182)\end{array}$ & $\begin{array}{c}\text { Both Dose } \\
(\mathrm{N}=26)\end{array}$ & $P$ value \\
\hline \multirow{5}{*}{ CRP } & $<6$ & $4(16.7 \%)$ & $50(27.5 \%)$ & $14(53.8 \%)$ & \multirow{5}{*}{$<0.001(\mathrm{~S})$} \\
\hline & $7-12$ & $6(25 \%)$ & $38(20.9 \%)$ & $5(19.2 \%)$ & \\
\hline & $13-18$ & $0(0 \%)$ & $30(16.5 \%)$ & $4(15.4 \%)$ & \\
\hline & $19-24$ & $6(25 \%)$ & $10(5.5 \%)$ & $1(3.8 \%)$ & \\
\hline & $>25$ & $8(33.3 \%)$ & $54(29.7 \%)$ & $2(7.7 \%)$ & \\
\hline \multirow{5}{*}{ D - dimer } & $<0.5$ & $10(41.7 \%)$ & $84(46.2 \%)$ & $6(23.1 \%)$ & \multirow{5}{*}{$0.035(\mathrm{~S})$} \\
\hline & $0.5-1$ & $7(29.2 \%)$ & $42(23.1 \%)$ & $15(57.7 \%)$ & \\
\hline & $1-1.5$ & $2(8.3 \%)$ & $22(12.1 \%)$ & $2(7.7 \%)$ & \\
\hline & $1.5-2$ & $3(12.5 \%)$ & $12(6.6 \%)$ & $2(7.7 \%)$ & \\
\hline & $>2$ & $4(16.7 \%)$ & $12(6.6 \%)$ & $1(3.8 \%)$ & \\
\hline \multirow{5}{*}{ IL-6 } & $<6$ & $6(25 \%)$ & $94(51.6 \%)$ & $8(30.8 \%)$ & \multirow{5}{*}{$0.001(\mathrm{~S})$} \\
\hline & $7-12$ & $5(20.8 \%)$ & $48(26.4 \%)$ & $14(53.8 \%)$ & \\
\hline & $13-18$ & $6(25 \%)$ & $11(6 \%)$ & $1(3.8 \%)$ & \\
\hline & $19-24$ & $3(12.5 \%)$ & $18(9.9 \%)$ & $2(7.7 \%)$ & \\
\hline & $\geq 25$ & $4(16.7 \%)$ & $11(6 \%)$ & $1(3.8 \%)$ & \\
\hline
\end{tabular}

\begin{tabular}{|c|c|c|c|c|}
\hline Outcome & $\begin{array}{l}\text { Unvaccinated } \\
(\mathrm{N}=24)\end{array}$ & $\begin{array}{c}\text { Single Dose } \\
(N=182)\end{array}$ & $\begin{array}{l}\text { Both Dose } \\
(\mathrm{N}=26)\end{array}$ & $P$ value \\
\hline $\begin{array}{c}\text { Survived } \\
\text { Died }\end{array}$ & $\begin{array}{c}22(91.7 \%) \\
2(8.3 \%) \\
\end{array}$ & $\begin{array}{c}180(98.9 \%) \\
2(1.1 \%)\end{array}$ & $\begin{array}{c}26(100 \%) \\
0(0 \%)\end{array}$ & $0.029(\mathrm{~S})$ \\
\hline \multicolumn{5}{|c|}{ Table 4. Final Outcome among Study Subjects } \\
\hline
\end{tabular}

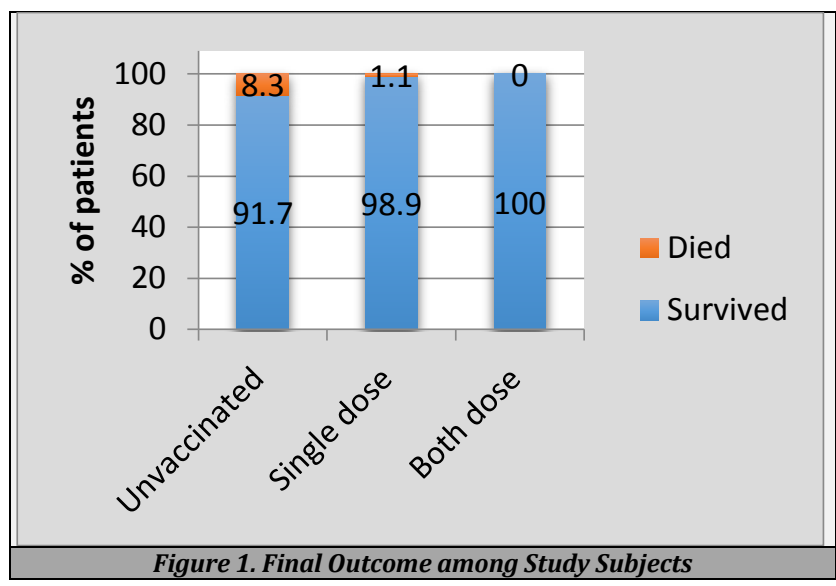

\section{DISCUSSION}

Vaccination against any communicable disease is the strongest weapon to control its spread, reduce severity of disease and ultimately its favourable impact on mortality, quality of health and economic losses. The world has seen tremendous preventive efficacy of small pox and polio vaccination which has been eliminated. Vaccination programme for prevention against various other viral diseases throughout the world has been a landmark for years together.

The present epidemic due to SARS-CoV-2 virus has been another viral disease with millions of fatalities in a short period of time. The world is now looking at mass vaccination against this virus with the hope to get rid of this pandemic. Most of the countries have now a vaccination drive with wide coverage. Various vaccines commercially available claim a protective efficacy ranging from 94-95 \% for fully vaccinated to $64 \%$ among partially vaccinated adults in preventing Covid-19 associated illness. ${ }^{26}$ An efficacy of $90 \%$ and $80 \%$ against SARS-CoV-2 has been reported for mRNA vaccine. ${ }^{27}$

The present study was carried out to evaluate comparative clinico-demographic profile of vaccinated, partially vaccinated and non-vaccinated individuals with RTPCR positive or clinico-epidemiologically suggestive of Covid19 pneumonias during second wave of this pandemic in India.
Our observations suggest that most of the unvaccinated study population belonged to less than 50 years of age as compared to partially or fully vaccinated study population. This difference was statistically significant and meant that the elderly population with preferential vaccination were more protected than their younger counterparts.

Cough, running nose and haemoptysis were more common symptoms among nonvaccinated population.

Major difference which could define the efficacy of partial or fully vaccination was CT severity score and rate of hospitalisation among these three groups. It is now well documented that CT severity score is an independent risk factor for prognosis of Covid pneumonias along with age and other biological markers. ${ }^{28}$ We could proudly conclude that $46.2 \%$ of vaccinated study population had CT severity score of less than 5 as compared to $23.1 \%$ and $8.3 \%$ respectively in partially vaccinated and unvaccinated study population. Similarly moderate disease on CT was much less in vaccinated population (38.5\%) as compared to $67.1 \%$ and $75 \%$ respectively in partially vaccinated and unvaccinated study population (table 2). This difference was statistically significant and probably proves the efficacy of vaccination in prevention of at least severe form of disease. Similar observations have been reported from Indian authors. ${ }^{29} \mathrm{~A}$ multivariate linear regression model showed that partial or fully vaccinated patients had lower CT severity score compared to unvaccinated patients. ${ }^{30}$ A statistically significant correlation between vaccination status and lung involvement based on HRCT thorax has also been reported in another Indian study.

Another significant parameter of efficacy of vaccination in our study was the need for hospitalisation. $92.3 \%$ of our vaccinated study population group were treated on OPD basis, rest needed only day care admission and none was hospitalised. Similar favourable results were also there for partially vaccinated study population. These figures were in sharp contrast for unvaccinated study population where 41.7 $\%$ required hospitalisation and $25 \%$ required day care facility treatment (Table 2).

Similar were the observations from community-based case control study performed in UK, where they found that vaccination was associated with reduced odds of hospitalisation or having more than five symptoms in the first week of illness following first or second dose of vaccination. Moreover, the risk for long Covid was also reduced. ${ }^{31}$

Our study also claims that biological markers of severity of Covid pneumonias (CRP, D-DIMER and IL-6) were also deranged to a lesser severity among vaccinated and partially vaccinated study population as compared to unvaccinated individuals. The difference was statistically significant (Table 3). These parameters closely followed the pattern observed for CT severity score and the need for hospitalisation. Although we did not have data for all the text book biomarkers for severity of Covid pneumonias, it has now been well documented that these three biomarkers along with others (low lymphocyte count, ferritin, cardiac troponin etc.) may be used in risk stratification to predict severe and fatal Covid-19 diseases in hospitalised patients. It is more likely that if some of these markers are altered than the course of the disease may unfavourable. ${ }^{32,33}$ 


\section{CONCLUSIONS}

Our study also reveals that both doses of vaccination provide $100 \%$ security against mortality. Overall, it is concluded that fully vaccinated individuals have a full proof protection against severe Covid infections irrespective of type of vaccination. It would further reduce long Covid as well as mortality due to this infection. A fast vaccination drive for younger people is warranted to further prevent the spread of infection.

\section{Limitations}

The sample size was small and we didn't classify our study population based on type of vaccination they received.

Data sharing statement provided by the authors is available with the full text of this article at jemds.com.

Financial or other competing interests: None.

Disclosure forms provided by the authors are available with the full text of this article at jemds.com.

\section{REFERENCES}

[1] \#IndiaFightsCorona Covid-19. MyGov.in. Govt of India.

[2] Dong E, Du H, Gardner L. An interactive web-based dashboard to track Covid-19 in real time. Lancet Infect Dis 2020;20(5):533-4.

[3] Chakraborty C, As Sharma AR, Bhattacharya M, et al. The current second wave and Covid-19 vaccination status in India. Brain Behav Immun 2021;96:1-4.

[4] Chakraborty C, Agoramoorthy G. India's cost-effective Covid-19 vaccine development initiatives. Vaccine 2020;38(50):7883-4.

[5] Bhattacharya A. Covid-19: active cases cross 1 million, at all-time high now. The Times of India. Retrieved 12 April 2021.

[6] Covid-19: India overtakes Brazil with second highest number of cases. The New Indian Express. PTI. 12 April 2021. Retrieved 22 April 2021.

[7] Jeffrey G, Sameer Y, Kumar $H$ et al. As Covid-19 Devastates India, Deaths Go Undercounted. The New York Times. ISSN 0362-4331, 24 April 2021.

[8] Julia H, McKeehan B, Kottasová I. India records more than 400,000 Covid-19 cases in a single day. CNN. 1 May 2021.

[9] Rogers K. Despite a billion vaccine shots given, Covid-19 runs rampant in much of the world. The New York Times 25 April 2021.

[10] Bagcchi S. The world's largest Covid-19 vaccination campaign. Lancet Infect Dis 2021;21(3):323.

[11] Kumar VM, Pandi-Perumal SR, Trakht I. et al. Strategy for Covid-19 vaccination in India: the country with the second highest population and number of cases. npj Vaccines 2021;60:1-7.

[12] Polack FP, Thomas SJ, Kitchin N, et al. Safety and efficacy of the BNT162b2 mRNA Covid-19 Vaccine. N Engl J Med 2020;383:2603-15.

[13] Singh AK, Phatak SR, Singh NK, et al. Antibody response after first-dose of ChAdOx1-nCOV (Covishield ${ }^{\mathrm{TM}}($ ) and BBV-152 (Covaxin ${ }^{\mathrm{TM}}($ ) ) amongst health care workers in
India: preliminary results of cross-sectional coronavirus vaccine-induced antibody titre (COVAT) study. medRxiv 2021.04.07.21255078

[14] World Health Organization. Hindustan Times. Covaxin efficacy $78 \%$ in Phase 3 analysis. May 29' 2021. https://www.hindustantimes.com/india-news/covaxinefficacy-78-in-phase-3-analysis-101622227104005.html

[15] Covid-19 Vaccines. Available from: https://www.who.int/emergencies/diseases/novelcoronavirus-2019/covid-19-vaccines.

[16] 2-4 per 10,000 people infected after getting vaccinated, Covaxin works against mutant viruses: Govt. Livemint. Available from: https://www.livemint.com/news/india/24-per-10-000people-infected-after-getting-vaccinated-govt11619022062884.html

[17] Zhang L, Jackson CB, Mou H, et al. The D614G Mutation in the SARS-CoV-2 Spike Protein Reduces S1 Shedding and Increases Infectivity. bioRxiv 2020; 2020.06.12.148726, doi:10.1101/2020.06.12.148726

[18] Yadav PD, Nyayanit DA, Sahay RR, et al. Isolation and Characterization of the New SARS-CoV-2 Variant in Travellers from the United Kingdom to India: VUI202012/01 of the B.1.1.7 Lineage. J Travel Med 2021;28:taab009. doi:10.1093/jtm/taab0

[19] Rambaut A, Holmes EC, O'Toole Á, et al. A dynamic nomenclature proposal for SARS-CoV-2 lineages to assist genomic epidemiology. Nat Microbiol 2020;5(11):14037.

[20] Kustin T, Harel N, Finkel U, et al. Evidence for increased breakthrough rates of SARS-CoV-2 variants of concern in BNT162b2-MRNA-vaccinated individuals. Nature Medicine 2021;27:1379-84.

[21] Hacisuleyman E, Hale C, Saito $Y$, et al. Vaccine Breakthrough Infections with SARS-CoV-2 Variants. N Engl J Med 2021;384(23):2212-8.

[22] CDC Covid-19 Vaccine Breakthrough Case Investigations Team. Covid-19 Vaccine Breakthrough Infections Reported to CDC - United States, January 1-April 30, 2021. MMWR Morb Mortal Wkly Rep 2021;70(21):7923.

[23] Loconsole D, Sallustio A, Accogli M, et al. Investigation of an outbreak of symptomatic SARS-CoV-2 VOC 202012/01-Lineage B.1.1.7 infection in healthcare workers, Italy. Clin Microbiol Infect Off Publ Eur Soc Clin Microbiol Infect Dis 2021;27(8):1174.e1-4.

[24] Philomina JB, Jolly B, John N, et al. Genomic survey of SARS-CoV-2 vaccine breakthrough infections in healthcare workers from Kerala, India. J Infect 2021;83(2):237-279.

[25] Tyagi K, Ghosh A, Nair D, et al. Breakthrough Covid19 infections after vaccinations in healthcare and other workers in a chronic care medical facility in New Delhi, India. Diabetes Metab Syndr 2021;15(3):1007-8.

[26] Yang R, Li X, Liu H, et al. Chest CT severity score: an imaging tool for assessing severe Covid-19. Radiol Cardiothorac Imaging 2020;30;2(2):e200047.

[27] Tenforde MW, Olson SM, Self WH, et al. Effectiveness of Pfizer-BioNTech and moderna vaccines against Covid-19 among hospitalised adults aged $>65$ years - United States, January-March 2021. MMWR Morb Mortal Wkly Rep 2021;70(18):674-9. 
[28] Abbasi B, Akhavan R, Khameneh AG, et al. Evaluation of the relationship between inpatient Covid-19 mortality and chest CT severity score. Am J Emerg Med 2021;45:458-63.

[29] Lakhia RT, Trivedi JR. The CT scan lung severity score and vaccination status in Covid-19 patients in India: perspective of an independent radiology practice. 2021 doi: https://doi.org/10.1101/2021.07.15.21260597

[30] Interim estimates of vaccine effectiveness of BNT162b2 and mRNA-1273 Covid-19 vaccines in preventing SARSCoV2 infection among health care personnel, first responders and other essential and frontline workersEight U. S. locations, December 2020-March 2021. MMWR 2021;70(13):495-500.
[31] Modi SD, Shah DS, Mundhra KS, et al. Comparative study of CT severity index and outcome in hospitalised vaccinated and nonvaccinated patients of Covid-19 pneumonia. J Radiol Clinc Imaging 2021;4(3):93-101.

[32] Michela A, Rose SP, Jordi M, et al. Risk factors and disease profile of post- vaccination SARS-CoV-2 infection in UK users of the Covid symptom study app: a prospective, community based, nested, case control study. Lancet Infect Dis 2022;22(1):43-55.

[33] Velavan TP, Meyer CG. Mild versus severe Covid-19: laboratory markers. Int J Infect Dis 2020;95:304-7. 